\title{
A Utilização da Calculadora Gráfica no ESTUdo do EquILíBrIo Acido/Base
}

\section{Mário Valente, * Helena Moreira}

Neste artigo exploram-se as potencialidades gráficas e de programação que as calculadoras gráficas, de uso obrigatório no ensino secundário, possuem. Essas potencialidades são aqui aplicadas ao estudo do equilíbrio ácido/base. Assim, são apresentados três pequenos programas que permitem visualizar graficamente as concentrações relativas das espécies envolvidas nas sucessivas protólises de ácidos mono, di e tripróticos, em função do $\mathrm{pH}$ do meio. É também apresentado um exemplo prático da sua utilização.

\section{INTRODUÇÃO}

Os programas das disciplinas de Física e Química A (nível 2) do $11^{\circ}$ ano (ou $12^{\circ}$ ano) e Química do $12^{\circ}$ ano de escolaridade [1] incluem o estudo do equilíbrio químico em geral e do equilíbrio ácido/base em particular. Assim, e tendo em conta a utilização obrigatória de calculadoras gráficas no ensino secundário, considerou-se de interesse a elaboração de três pequenos programas que permitissem uma fácil visualização da variação das concentrações relativas das espécies envolvidas nas protólises de ácidos mono, di e tripróticos, em função do $\mathrm{pH}$ do meio.

Os equilíbrios de protólise são traduzidos pelas equações químicas no quadro abaixo.

Da substituição das expressões das constantes de equilíbrio na expressão do balanço de massa total correspondente ao tipo de ácido:

a) para um ácido monoprótico $(\mathrm{HA})$ :

$$
\mathrm{HA}(\mathrm{aq})+\mathrm{H}_{2} \mathrm{O}(\mathrm{l}) \leftrightarrows \mathrm{A}^{-}(\mathrm{aq})+\mathrm{H}_{3} \mathrm{O}^{+}(\mathrm{aq})
$$

b) para um ácido diprótico $\left(\mathrm{H}_{2} \mathrm{~A}\right)$ :

$$
\begin{aligned}
& \mathrm{H}_{2} \mathrm{~A}(\mathrm{aq})+\mathrm{H}_{2} \mathrm{O}(\mathrm{l}) \leftrightarrows \mathrm{HA}^{-}(\mathrm{aq})+\mathrm{H}_{3} \mathrm{O}^{+}(\mathrm{aq}) \\
& \mathrm{HA}^{-}(\mathrm{aq})+\mathrm{H}_{2} \mathrm{O}(\mathrm{l}) \leftrightarrows \mathrm{A}^{2-}(\mathrm{aq})+\mathrm{H}_{3} \mathrm{O}^{+}(\mathrm{aq})
\end{aligned}
$$

c) e para um ácido triprótico $\left(\mathrm{H}_{3} \mathrm{~A}\right)$ :

$$
\begin{array}{ll}
\mathrm{H}_{3} \mathrm{~A}(\mathrm{aq})+\mathrm{H}_{2} \mathrm{O}(\mathrm{l}) \leftrightarrows \mathrm{H}_{2} \mathrm{~A}^{-}(\mathrm{aq})+\mathrm{H}_{3} \mathrm{O}^{+}(\mathrm{aq}) & \left(K_{\mathrm{a} 1}\right) \\
\mathrm{H}_{2} \mathrm{~A}^{-}(\mathrm{aq})+\mathrm{H}_{2} \mathrm{O}(\mathrm{l}) \leftrightarrows \mathrm{HA}^{2^{-}}(\mathrm{aq})+\mathrm{H}_{3} \mathrm{O}^{+}(\mathrm{aq}) & \left(K_{\mathrm{a} 2}\right) \\
\mathrm{HA}^{2-}(\mathrm{aq})+\mathrm{H}_{2} \mathrm{O}(\mathrm{l}) \leftrightarrows \mathrm{A}^{3-}(\mathrm{aq})+\mathrm{H}_{3} \mathrm{O}^{+}(\mathrm{aq}) & \left(K_{\mathrm{a} 3}\right)
\end{array}
$$

Colégio D. Duarte, Rua Visconde de Setúbal, 86, 4200-497 Porto, Portugal madmage1@yahoo.com
$\frac{\left[\mathrm{H}_{3} \mathrm{~A}\right]}{\left[\mathrm{H}_{3} \mathrm{~A}\right]_{\text {rotal }}}=\left(\frac{\mathrm{K}_{\mathrm{a} 1} \cdot \mathrm{K}_{\mathrm{a2}} \cdot \mathrm{K}_{\mathrm{a}}}{\left[\mathrm{H}_{3} \mathrm{O}^{+}\right]^{\beta}}+\frac{\mathrm{K}_{\mathrm{a}} \cdot \mathrm{K}_{\mathrm{a2}}}{\left[\mathrm{H}_{3} \mathrm{O}^{+}\right]^{2}}+\frac{\mathrm{K}_{\mathrm{a} 1}}{\left[\mathrm{H}_{3} \mathrm{O}^{+}\right]^{+1}}\right)^{-1}$

$\frac{\left[\mathrm{H}_{2} \mathrm{~A}\right]}{\left[\mathrm{H}_{3} \mathrm{~A}\right]_{\text {rotal }}}=\left(\frac{\mathrm{K}_{22} \cdot \mathrm{K}_{23}}{\left[\mathrm{H}_{3} \mathrm{O}^{+}\right]^{2}+}+\frac{\mathrm{K}_{22}}{\left[\mathrm{H}_{3} \mathrm{O}^{+}\right]}+1+\frac{\left[\mathrm{H}_{3} \mathrm{O}^{+}\right]}{\mathrm{K}_{\mathrm{al}}}\right)^{-1}$

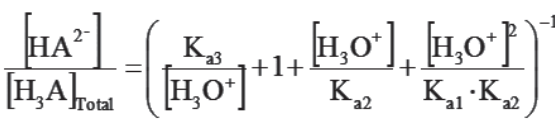

$\frac{\left[\mathrm{A}^{3}\right]}{\left[\mathrm{H}_{3} \mathrm{~A}\right]_{\text {rotal }}}=\left(1+\frac{\left[\mathrm{H}_{3} \mathrm{O}^{+}\right]}{\left.\mathrm{K}_{\mathrm{a}}\right]^{3}}+\frac{\left[\mathrm{H}_{3} \mathrm{O}^{+}\right]^{\mathrm{P}}}{\mathrm{K}_{\mathrm{a2}} \cdot \mathrm{K}_{\mathrm{a3}}}+\frac{\left[\mathrm{H}_{3} \mathrm{O}^{+}\right]^{\beta}}{\mathrm{K}_{\mathrm{al}} \cdot \mathrm{K}_{\mathrm{a2}} \cdot \mathrm{K}_{\mathrm{az}}}\right)^{-1}$

que traduzem as variações das concentrações relativas das várias espécies envolvidas nas sucessivas protólises, em função da concentração do meio em catião hidrónio $\left(\mathrm{H}_{3} \mathrm{O}^{+}\right)$.

\section{Programação da máQuina de CALCULAR}

Nos três programas a seguir apresentados é usada a linguagem própria das calculadoras gráficas Texas. No entanto, estes programas podem ser facilmente convertidos para as linguagens características de outras máquinas como, por exemplo, as calculadoras gráficas CASIO.

No caso dos ácidos monopróticos o programa começa por requerer a introdução do valor da constante de acidez, $K_{a 1}$, para de seguida criar duas funções $\left(Y_{1}\right.$ e $\left.Y_{2}\right)$ que correspondem às variações as razões $[\mathrm{HA}] /[\mathrm{HA}]_{\text {total }}$ e $\left[\mathrm{A}^{-}\right] /[\mathrm{HA}]_{\text {total }}$, em função do valor de $\mathrm{pH}$ do meio (usando a conversão: $\left.\left[\mathrm{H}_{3} \mathrm{O}^{+}\right]=10^{-\mathrm{pH}}\right)$. Por fim, o programa executa a representação gráfica das funções criadas. 


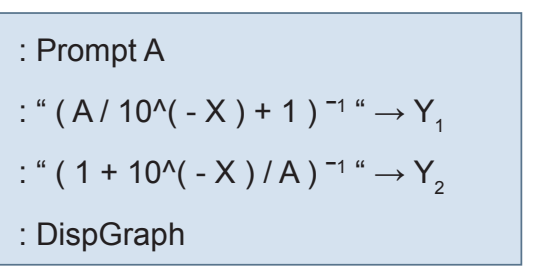

É importante definir a janela de visualização para que a representação gráfica gerada permita uma leitura com mais sentido. Para o efeito, sugerem-se os seguintes valores a introduzir na página window: $X \min =0 ; X \max =$ $14 ; X s c l=1 ; Y \min =-0,2 ; Y \max =1$; $Y s c l=1 ; X r e s=1$.

Para ácidos dipróticos o programa inicia por requerer a introdução dos valores das constantes de acidez, $K_{a 1}$ e $K_{\mathrm{a}}$, para de seguida criar três funções $\left(Y_{1}, Y_{2}\right.$ e $\left.Y_{3}\right)$ que correspondem às variações das razões $\left[\mathrm{H}_{2} \mathrm{~A}\right] /\left[\mathrm{H}_{2} \mathrm{~A}\right]_{\text {total }}$, $\left[\mathrm{HA}^{-}\right] /\left[\mathrm{H}_{2} \mathrm{~A}\right]_{\text {total }}$ e $\left[\mathrm{A}^{2-}\right] /\left[\mathrm{H}_{2} \mathrm{~A}\right]_{\text {total' }}$, em função do valor de $\mathrm{pH}$ do meio. constantes de protólise, a $25^{\circ} \mathrm{C}$, são apresentadas na Tabela 1.

Tabela 1 Valores das constantes de protólise, a
$25{ }^{\circ} \mathrm{C}$, de três ácidos com enxofre $[2,3]$
\begin{tabular}{|l|c|c|}
\hline & $K_{\mathrm{a} 1}$ & $K_{\mathrm{a} 2}$ \\
\hline $\mathrm{H}_{2} \mathrm{SO}_{4}$ & $3,7 \times 10^{8}$ & $1,2 \times 10^{-2}$ \\
\hline $\mathrm{H}_{2} \mathrm{SO}_{3}$ & $1,2 \times 10^{-2}$ & $6,2 \times 10^{-8}$ \\
\hline $\mathrm{H}_{2} \mathrm{~S}$ & $8,9 \times 10^{-8}$ & $1,2 \times 10^{-13}$ \\
\hline
\end{tabular}

A introdução dos valores das constantes de protólise no programa correspondente resulta nas representações gráficas apresentadas na Fig. 1.
Para o ácido sulfídrico (Fig. $1 \mathrm{C}$ ) a espécie dominante até $\mathrm{pH} \approx 7$ é $\mathrm{H}_{2} \mathrm{~S}$ (com uma protólise muito incompleta em grande parte da escala de $\mathrm{pH}$, este é um ácido muito fraco). Entre $\mathrm{pH} \approx 7$ e $\mathrm{pH} \approx 13$ domina a espécie $\mathrm{HS}^{-}$e a partir de $\mathrm{pH} \approx 13$ domina a espécie $\mathrm{S}^{2-}$.

Conclui-se que é possível ter soluções aquosas de sulfato de sódio (ou de qualquer outro catião inerte) a $\mathrm{pH}$ neutro, mas isso já não é possível para soluções de sulfito, e muito me-

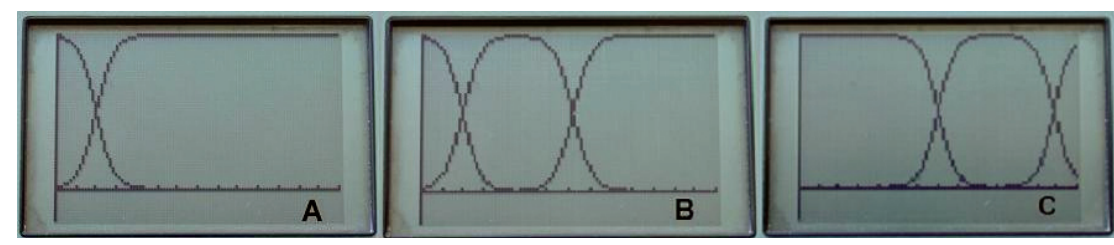

Figura 1 Representações gráficas das concentrações relativas das várias espécies resultantes das protólises sucessivas dos ácidos $\mathrm{H}_{2} \mathrm{SO}_{4}(\mathrm{~A}), \mathrm{H}_{2} \mathrm{SO}_{3}$ (B) e $\mathrm{H}_{2} \mathrm{~S}(\mathrm{C})$, em função do $\mathrm{pH}$ do meio (intervalo de $\mathrm{pH}$ de $\mathrm{O}$ a 14)

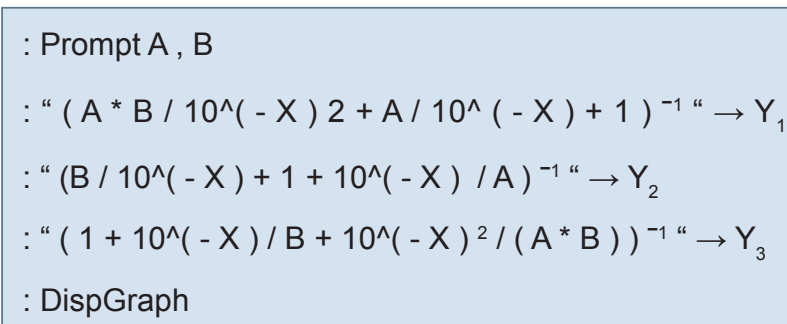

No caso dos ácidos tripróticos o programa requer a introdução dos valores das constantes de acidez, $K_{a 1}, K_{a 2}$ e $K_{a 3}$, para de seguida criar quatro funções $\left(Y_{1}, Y_{2}, Y_{3}\right.$ e $\left.Y_{4}\right)$ que correspondem às variações das razões $\left[\mathrm{H}_{3} \mathrm{~A}\right] /\left[\mathrm{H}_{3} \mathrm{~A}\right]_{\text {total' }},\left[\mathrm{H}_{2} \mathrm{~A}^{-}\right] /\left[\mathrm{H}_{3} \mathrm{~A}\right]_{\text {total' }}$, $\left[\mathrm{HA}^{2-}\right] /\left[\mathrm{H}_{3} \mathrm{~A}\right]_{\text {total }}$ e $\left[\mathrm{A}^{3-}\right] /\left[\mathrm{H}_{3} \mathrm{~A}\right]_{\text {total }}$, em função do valor de $\mathrm{pH}$ do meio.

Pode-se observar (Fig.1 A) que no caso do ácido sulfúrico o valor de $K_{a 1}$ é tão elevado que mesmo a $\mathrm{pH}=0,0$ não existe praticamente $\mathrm{H}_{2} \mathrm{SO}_{4}$ (com a sua protólise praticamente completa, este é um ácido muito forte), sendo $\mathrm{HSO}_{4}^{-}$a espécie maioritariamente presente até um $\mathrm{pH} \approx 2$, a partir desse valor a

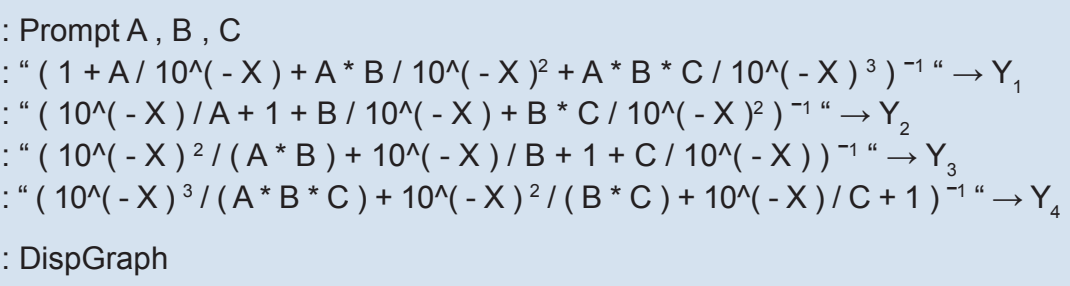

UM EXEMPLO - TRÊS ÁCIDOS COM ENXOFRE

Comparam-se de seguida as protólises de três ácidos dipróticos contendo enxofre: o ácido sulfúrico $\left(\mathrm{H}_{2} \mathrm{SO}_{4}\right)$, o ácido sulfuroso $\left(\mathrm{H}_{2} \mathrm{SO}_{3}\right)$ e $\mathrm{O}$ ácido sulfídrico $\left(\mathrm{H}_{2} \mathrm{~S}\right)$. As correspondentes espécie dominante passa a ser $\mathrm{SO}_{4}{ }^{2-}$.

No caso do ácido sulfuroso (Fig. 1 B) é a espécie $\mathrm{H}_{2} \mathrm{SO}_{3}$ que domina até um $\mathrm{pH} \approx 2$. Entre $\mathrm{pH} \approx 2$ e $\mathrm{pH} \approx 7$ a espécie dominante é $\mathrm{HSO}_{3}^{-}$. A partir de $\mathrm{pH} \approx 7$, domina a espécie $\mathrm{SO}_{3}{ }^{2-}$. nos de sulfureto de sódio, pois estas apresentam carácter alcalino.

Verifica-se também que uma solução diluída de hidrogenossulfato de sódio em água apresentará um $\mathrm{pH}$ bem mais baixo que uma solução de hidrogenossulfito de sódio, mas ambas apresentam ainda um carácter ácido. Uma solução aquosa de hidrogenossulfureto de sódio já apresentará um carácter alcalino.

Conclui-se também que em soluções equivalentes dos mesmos sais, mas agora em solução aquosa de ácido clorídrico $0,1 \mathrm{~mol} / \mathrm{dm}^{3}$ (cujo $\mathrm{pH} \approx 1$ ), o ião hidrogenossulfato permanece o ião dominante. O ião hidrogenossulfito é maioritariamente convertido em ácido sulfuroso e o ião hidrogenossulfureto é completamente convertido em ácido sulfídrico.

É também possível verificar que adicionando um pouco de um ácido a uma solução aquosa de hidrogenossulfito de sódio a $\mathrm{pH}=5$, podemos ver pelo gráfico correspondente (Fig. $1 \mathrm{~B}$ ) que não se produzirá alteração significativa de concentração desse ião, pelo que o ião $\mathrm{H}_{3} \mathrm{O}^{+}$adicionado não vai ser significativamente usado num processo ácido-base, causando consequentemente uma variação de $\mathrm{pH}$ 
significativa. Já a mesma quantidade de ião $\mathrm{H}_{3} \mathrm{O}^{+}$adicionada a uma solução contendo os iões hidrogenossulfito e sulfito, a $\mathrm{pH}=7$, não vai provocar alteração significativa de $\mathrm{pH}$ (efeito tampão) pois será maioritariamente consumida na conversão de ião sulfito a ião hidrogenossulfito.

\section{Conclusão}

Autilização da calculadora gráfica pode apresentar muitos benefícios se as suas capacidades forem devidamente exploradas. De facto é importante fazer notar aos alunos que esses benefícios vão muito além da sua simples utilização como formulário ou auxiliar de memória, para testes e exames.

Se devidamente "trabalhados" com exemplos sugestivos, os programas apresentados podem ser úteis não só no estudo do equilíbrio ácido/base como também para a criação, nos alunos, de noções de programação e para uma melhoria das suas capacidades de visualização gráfica.

\section{Notas}

1 Com o fim de facilitar a introdução dos programas na calculadora a quem não tenha muita prática, descrevem-se os caminhos para chegar aos comandos menos usuais: Faz-se uso do comando "Prompt', obtido digitando a tecla "PRGM", escoIhendo a opção "I/O" e digitando a tecla "2"; da função "10^^", obtida digitando as duas teclas "2nd" e "LOG"; da seta " $\rightarrow$ " obtida digitando a tecla "STO" e das funções $Y_{n}$, obtidas digitando a tecla "VARS", escolhendo a opção "Y-VARS" e digitando a tecla "1".
2 É possível obter os valores numéricos das concentrações relativas digitando as teclas "2nd", "TRACE" e "VALUE", digitando, de seguida, o valor do $\mathrm{pH}$ para o qual se pretendem as concentrações. Altera-se a função premindo as teclas cima ou baixo.

\section{REFERÊNCIAS}

[1] Programas da disciplina de Física e Química A (nível 2) $11^{\circ}$ ou $12^{\circ}$ anos e de Química do $12^{\circ}$ ano:

http://www.dgidc.min-edu.pt/programs/prog_eg.asp

[2] P.W. Atkins, Physical Chemistry, $3^{\text {a }}$ edição, Oxford University Press, Oxónia, 1989

[3] Tabelas e Formulário de Química, Departamento de Química, Universidade do Porto.

\section{Actualidades Científicas}

\section{UMA ECONOMIA DO HIDROGÉNIO ECONÓMICA}

Possivelmente, o hidrogénio será um dos mais importantes combustíveis do futuro. No entanto, para se conseguir atingir a chamada economia do hidrogénio viável, os investigadores da área energética deverão desenvolver métodos eficientes de produção do gás a partir de fontes renováveis de energia, como a solar. Por exemplo, não há qualquer vantagem em utilizar fontes fósseis para produzir hidrogénio para veículos alternativos, para além de possíveis benefícios na redução dos níveis locais de poluição.

Assim, investigadores do Max Planck Institute, na Alemanha, em colaboração com colegas noruegueses e norte-americanos, desenvolveram um catalisador que pode converter a água directamente em hidrogénio por acção da luz solar, através de um processo análogo ao da fotossíntese de hidratos de carbono que ocorre nas plantas, que usa dióxido de carbono e água como matérias primas na presença de luz solar.

O novo catalisador semicondutor, produzido por Martin Demuth (do Max Planck Institute for Bioinorganic Chemistry, em Mulheim an der Ruhr) e seus colaboradores, não só produz hidrogénio, como constitui um meio para o seu armazenamento, o que significa uma vantagem adicional, já que contribui para facilitar a separação do hidrogénio do outro produto da reacção, o oxigénio.

Demuth explica que: "A geração de hidrogénio e oxigénio a partir da água através de semicondutores é uma contribuição importante para a aplicação da energia solar.

Os semicondutores adequados para utilização como fotocatalisadores têm sido difíceis de sintetizar, apresentam características de absorção de luz desfavoráveis, ou decompõem-se durante a reacção."
Para ultrapassar estes múltiplos problemas, Demuth e a sua equipa recorreram ao dissiliceto de titânio $\left(\mathrm{TiSi}_{2}\right)$. Este material apresenta propriedades optoelectrónicas ideais para o seu uso como captador de energia solar. Para além disso, absorve numa ampla gama do espectro solar, é fácil de produzir e é barato. (adaptado de webzine Reactive Reports 69, 2007). 